\title{
SHORT REPORT \\ Pregnant with HIV before age 25: data from a large national study in Italy, 2001-2016
}

\author{
M. FLORIDIA ${ }^{1}$, G. MASUELLI ${ }^{2}$, E. TAMBURRINI ${ }^{3}$, I. CETIN $^{4}$, G. LIUZZI $^{5}$, \\ P. MARTINELLI ${ }^{6}$, G. GUARALDI ${ }^{7}$, A. SPINILLO ${ }^{8}$, A. VIMERCATI ${ }^{9}$, \\ G. MASO ${ }^{10}$, C. PINNETTI ${ }^{5}$, V. FRISINA ${ }^{2}$, S. DALZERO ${ }^{11}$, M. RAVIZZA ${ }^{11}$ \\ on behalf of The Italian Group on Surveillance on Antiretroviral Treatment in Pregnancy $\dagger$ \\ ${ }^{1}$ National Centre for Global Health, Istituto Superiore di Sanità, Rome, Italy \\ ${ }^{2}$ Department of Obstetrics and Neonatology, Città della Salute e della Scienza Hospital, and University of Turin, \\ Italy \\ ${ }^{3}$ Department of Infectious Diseases, Catholic University, Rome, Italy \\ ${ }_{5}^{4}$ Department of Obstetrics and Gynaecology, Luigi Sacco Hospital and University of Milan, Italy \\ ${ }^{5}$ I.N.M.I. Lazzaro Spallanzani, Rome, Italy \\ ${ }^{6}$ Department of Neurosciences, Reproductive and Dentistry Science, University Federico II, Naples, Italy \\ ${ }^{7}$ Department of Medical Specialties, Infectious Diseases Clinic, University of Modena and Reggio Emilia, \\ Modena, Italy \\ ${ }^{8}$ Department of Obstetrics and Gynaecology, IRCCS S. Matteo, Pavia, Italy \\ ${ }^{9}$ Department of Obstetrics and Gynaecology, University of Bari, Italy \\ ${ }^{10}$ Institute for Maternal and Child Health, IRCCS Burlo Garofolo, Trieste, Italy \\ ${ }^{11}$ Department of Obstetrics and Gynaecology, DMSD San Paolo Hospital Medical School, University of Milan, \\ Italy
}

Received 28 March 2017; Final revision 17 May 2017; Accepted 6 June 2017

\section{SUMMARY}

Young pregnant women with HIV may be at significant risk of unplanned pregnancy, lower treatment coverage, and other adverse pregnancy outcomes. In a large cohort of pregnant women with HIV in Italy, among 2979 pregnancies followed in 2001-2016, 9.0\% were in women $<25$ years, with a significant increase over time (2001-2005: 7.0\%; 2006-2010: 9.1\%; 2011-2016: $12 \cdot 2 \%, P<0 \cdot 001)$. Younger women had a lower rate of planned pregnancy $(23 \cdot 2 \%$ vs. $37 \cdot 7 \%$, odds ratio (OR) $0 \cdot 50,95 \%$ confidence interval (CI) $0 \cdot 36-0 \cdot 69$ ), were more frequently diagnosed with HIV in pregnancy $(46 \cdot 5 \%$ vs. $20 \cdot 9 \%$, OR $3 \cdot 29,95 \%$ CI $2 \cdot 54-4 \cdot 25)$, and, if already diagnosed

\footnotetext{
* Author for correspondence: M. Floridia, National Center for Global Health, Istituto Superiore di Sanità, Viale Regina Elena 299,00161 Rome, Italy.

(Email: marco.floridia@iss.it)

The Italian Group on Surveillance of Antiretroviral Treatment in Pregnancy:

Project coordinators: M. Floridia, M. Ravizza, E. Tamburrini.

Participants: M. Ravizza, E. Tamburrini, F. Di Lorenzo, G. Sterrantino, M. Meli, I. Campolmi, F. Vichi, B. Del Pin, R. Marocco, C. Mastroianni, V.S. Mercurio, A. Maccabruni, M. Zaramella, B. Mariani, G. Guaraldi, G. Nardini, C. Stentarelli, B. Beghetto, A.M. Degli Antoni, A. Molinari, M.P. Crisalli, A. Donisi, M. Piepoli, V. Cerri, G. Zuccotti, V. Giacomet, S. Coletto, F. Di Nello, C. Madia, G. Placido, P. Milini, F. Savalli, V. Portelli, F. Sabbatini, D. Francisci, G. Angeli, L. Bernini, P. Grossi, L. Rizzi, M. Bernardon, G. Maso, E.Rizzante, C.Belcaro, A. Meloni, M. Dedoni, F. Ortu, P. Piano, A. Citernesi, I. Bordoni Vicini, K. Luzi, A. Spinillo, M. Roccio, A. Vimercati, A. Miccolis, A. De Gennaro, B. Guerra, F. Cervi, G. Simonazzi, E. Margarito, M.G. Capretti, C. Marsico, G. Faldella, M. Sansone, P. Martinelli, A. Agangi, A. Capone, G.M. Maruotti, C. Tibaldi, L. Trentini, T. Todros, G. Masuelli, V. Frisina, I. Cetin, T. Brambilla, V. Savasi, C. Personeni, C. Giaquinto, M. Fiscon, E. Rubino, L. Franceschetti, B. Tassis, O. Genovese, C. Cafforio, C. Pinnetti, G. Liuzzi, A.M. Casadei, A.F. Cavaliere, M. Cellini, A.M. Marconi, S. Dalzero, V. Sacchi, M. Ierardi, C. Polizzi, A. Mattei, M.F. Pirillo, R. Amici, C.M. Galluzzo, S. Donnini, S. Baroncelli, M. Floridia. Advisory Board: A. Cerioli, M. De Martino, F. Parazzini, E. Tamburrini, S. Vella.

SIGO-HIV Group National Coordinators: P. Martinelli, M. Ravizza.
} 
with HIV before pregnancy, were less frequently on antiretroviral treatment at conception $(<25$ years: $56 \cdot 3 \%$; $\geqslant 25$ years: $69 \cdot 0 \%$, OR $0 \cdot 58,95 \%$ CI $0 \cdot 41-0 \cdot 81)$. During pregnancy, treatment coverage was almost universal in both age groups (98.5\% vs. 99.3\%), with no differences in rate of HIV viral suppression at third trimester and adverse pregnancy outcomes. The data show that young women represent a growing proportion of pregnant women with HIV, and are significantly more likely to have unplanned pregnancy, undiagnosed HIV infection, and lower treatment coverage at conception. During pregnancy, antiretroviral treatment, HIV suppression, and pregnancy outcomes are similar compared with older women. Earlier intervention strategies may provide additional benefits in the quality of care for women with HIV.

Key words: Antiretroviral treatment, HIV diagnosis, HIV testing, pregnancy, women's health.

\section{INTRODUCTION}

Young women (15-24 years) are particularly vulnerable to sexually transmitted infections and HIV [1]. In this particular population, pregnancy is commonly unplanned and undesired, and quite frequently occurs in women who had never been tested for HIV [1, 2]. Age, lower therapeutic adherence, and a combination of socio-economic factors pose young pregnant women with HIV at risk of adverse pregnancy outcomes, lower treatment coverage, less stringent suppression of HIV, and vertical transmission [3]. It is therefore of importance to better define the prevalence of undiagnosed HIV infection, unplanned pregnancy, adverse pregnancy outcomes and suboptimal HIV suppression in this fragile population. To address this issue, we used data from a large national cohort of pregnant women with HIV and explored temporal trends in the proportion of women with HIV with pregnancy at young age ( $<25$ years), and assessed in this specific group the rates of planned pregnancy, HIV diagnosis in pregnancy, antiretroviral (ARV) treatment at conception and during pregnancy, HIV viral suppression at third trimester, HIV vertical transmission, and main pregnancy outcomes.

\section{METHODS}

Data from the Italian National Program on Surveillance on Antiretroviral Treatment in Pregnancy, an ongoing observational study established in Italy in 2001, were used [4]. Only HIV-positive pregnant women are eligible for the study, and treatment of HIV infection is decided by the treating physician, usually according to national guidelines. Women provide consent based on a patient information sheet approved by the competent Ethics Committee (deliberation 578, 28 September 2001, I.N. M.I. Lazzaro Spallanzani Ethics Committee, Rome).
For the current analysis, all pregnancies with known maternal age and available date of HIV diagnosis were considered eligible. The study period (2001-2016) was divided in three intervals of 5 years each (20012005, 2006-2010, 2011-2016), and temporal trends were analyzed using the $\chi^{2}$ test for trend. Other categorical variables were compared using the $\chi^{2}$ test, with odds ratios (ORs) and 95\% confidence intervals (CIs) calculated. In order to adjust for potential confounders, also defined in previous analyses [4, 5], the role of young age as predictor of unplanned pregnancy and undiagnosed HIV infection was further evaluated in multivariable logistic regression analyses. Gender- and gestational age-adjusted $Z$-scores for birthweight were calculated according to recent national references [6]. Major birth defects were defined according to the Antiretroviral Pregnancy Registry definition [7]. For all analyses, $P$ values $<0.05$ were considered significant. All statistical analyses were performed with the SPSS software, version 22 (IBM Corp, Released 2013, Armonk, New York, USA).

\section{RESULTS}

As of 24 February 2017, 2979 pregnancies had available information and were included in the analysis. The median age at entry in pregnancy was 33 years (interquartile range 29-36), with $9 \cdot 0 \%$ (269/2979) of the women $<25$ of age. Younger $(<25$ years) and older ( $\geqslant 25$ years) had identical mean CD4 count levels at entry $\left(493 / \mathrm{mm}^{3}\right.$ in younger women vs. 494/ $\mathrm{mm}^{3}$ in older women, $P=0.966$ ), but younger women were more frequently of foreign origin (68.2\% vs. $45 \cdot 6 \%$, OR $2 \cdot 56,95 \%$ CI $1 \cdot 94-3 \cdot 37, P<$ $0 \cdot 001)$. Only a small proportion of pregnancies were planned $(925 / 2540,36 \cdot 4 \%)$, with a significantly lower probability of planned pregnancy among younger women $(23 \cdot 2 \% \quad$ vs. $37 \cdot 7 \%$, OR $0 \cdot 50, \quad 95 \%$ CI 
Table 1. Temporal trends (2001-2016) in pregnancy planning, diagnosis of HIV in pregnancy, and treatment coverage in mothers and infants

\begin{tabular}{|c|c|c|c|c|c|}
\hline & 2001-2016 & 2001-2005 & 2006-2010 & 2011-2016 & $P$ value* \\
\hline Age lower than $25(\%)$ & $9 \cdot 0(269 / 2979)$ & $7 \cdot 0(82 / 1170)$ & $9 \cdot 1(98 / 1079)$ & $12 \cdot 2(89 / 730)$ & $<0 \cdot 001$ \\
\hline \multicolumn{6}{|l|}{ Pregnancy planned (\%): } \\
\hline Overall & $36 \cdot 4(925 / 2540)$ & $34 \cdot 3(346 / 1009)$ & $36 \cdot 7(338 / 922)$ & $39 \cdot 6(241 / 609)$ & $0 \cdot 032$ \\
\hline Age lower than 25 & $23 \cdot 2(51 / 220)$ & $17 \cdot 9(12 / 67)$ & $23 \cdot 2(19 / 82)$ & $28 \cdot 2(20 / 71)$ & $0 \cdot 154$ \\
\hline Age 25 or older & $37 \cdot 7(874 / 2320)$ & $35 \cdot 5(334 / 942)$ & $38 \cdot 0(319 / 840)$ & $41 \cdot 1(221 / 538)$ & 0.031 \\
\hline \multicolumn{6}{|c|}{ Diagnosis of HIV in current pregnancy (\%): } \\
\hline Overall & $23 \cdot 2(691 / 2979)$ & $23 \cdot 0(269 / 1170)$ & $24 \cdot 4(263 / 1079)$ & $21 \cdot 8(159 / 730)$ & $0 \cdot 661$ \\
\hline Age lower than 25 & $46 \cdot 5(125 / 269)$ & $51 \cdot 2(42 / 82)$ & $44 \cdot 9(44 / 98)$ & $43 \cdot 8(39 / 89)$ & $0 \cdot 338$ \\
\hline Age 25 or older & $20 \cdot 9(566 / 2710)$ & $20 \cdot 9(227 / 1088)$ & $22 \cdot 3(219 / 981)$ & $18 \cdot 7(120 / 641)$ & $0 \cdot 410$ \\
\hline \multicolumn{6}{|c|}{ Mothers on treatment at conception ${ }^{\dagger}(\%)$ : } \\
\hline Overall & $68 \cdot 2(1553 / 2277)$ & $61.9(555 / 897)$ & $66 \cdot 9(543 / 812)$ & $80 \cdot 1(455 / 568)$ & $<0.001$ \\
\hline Age lower than 25 & $56 \cdot 3(81 / 144)$ & $42 \cdot 5(17 / 40)$ & $55 \cdot 6(30 / 54)$ & $68 \cdot 0(34 / 50)$ & $0 \cdot 016$ \\
\hline Age 25 or older & $69 \cdot 0(1472 / 2133)$ & $62 \cdot 8(538 / 857)$ & $67 \cdot 7(513 / 758)$ & $81 \cdot 3(421 / 518)$ & $<0 \cdot 001$ \\
\hline \multicolumn{6}{|c|}{ Mothers on treatment in pregnancy $(\%)$ : } \\
\hline Overall & $96 \cdot 5(2784 / 2885)$ & $95 \cdot 6(1093 / 1143)$ & $96 \cdot 6(1009 / 1044)$ & $97 \cdot 7(682 / 698)$ & $0 \cdot 017$ \\
\hline Age lower than 25 & $94 \cdot 7(248 / 262)$ & $93 \cdot 8(76 / 81)$ & $97 \cdot 9(92 / 94)$ & $92 \cdot 0(80 / 87)$ & 0.565 \\
\hline Age 25 or older & $96 \cdot 7(2536 / 2623)$ & $95 \cdot 8(1017 / 1062)$ & $96 \cdot 5(917 / 950)$ & $98 \cdot 5(602 / 611)$ & 0.003 \\
\hline \multicolumn{6}{|l|}{ Infant prophylaxis (\%): } \\
\hline Overall & $97 \cdot 3(1967 / 2021)$ & $95 \cdot 8(789 / 824)$ & $97 \cdot 8(719 / 735)$ & $99 \cdot 4(459 / 462)$ & $<0.001$ \\
\hline Age lower than 25 & $97 \cdot 2(172 / 177)$ & $96 \cdot 6(56 / 58)$ & $96 \cdot 9(62 / 64)$ & $98 \cdot 2(54 / 55)$ & 0.604 \\
\hline Age 25 or older & $97 \cdot 3(1795 / 1844)$ & $95 \cdot 7(733 / 766)$ & $97 \cdot 9(657 / 671)$ & $99 \cdot 5(405 / 407)$ & $<0.001$ \\
\hline
\end{tabular}

$* \chi^{2}$ for trend.

$\dagger$ Women with HIV infection diagnosed before pregnancy only.

$0 \cdot 36-0.69, P<0 \cdot 001)$. The main temporal trends in pregnancy planning, diagnosis of HIV in pregnancy, and ARV treatment coverage in mothers and infants are summarized in Table 1. The proportion of young women increased significantly over time, from $7 \cdot 0 \%$ in $2001-2005$ to $12 \cdot 2 \%$ in $2011-2016$. The proportion of planned pregnancy also increased over time, but this increase was statistically significant only in the entire group and in the subgroup of older women. The rate of HIV diagnosis in current pregnancy did not change over time, but young women were significantly more likely $(46.5 \%$ compared with $20.9 \%$ in older women) to have HIV diagnosed in pregnancy (OR $3 \cdot 29,95 \%$ CI $2 \cdot 54-4 \cdot 25, P<$ $0 \cdot 001$ ). Among the women with HIV already known, overall treatment coverage at conception was $68 \cdot 2 \%$, with a significant increase in recent years, from $61.9 \%$ in $2001-2005$ to $80 \cdot 1 \%$ in $2011-2016$ ( $P<$ $0 \cdot 001)$. This significant temporal trend was present in both age groups (Table 1), but younger women were significantly less likely to be on treatment at conception (overall: $56 \cdot 3 \%$ vs. $69 \cdot 0 \%$, OR $0 \cdot 58,95 \%$ CI $0 \cdot 45-0 \cdot 81, P=0 \cdot 002)$. Subsequent treatment coverage in pregnancy was almost universal, with ARV treatment administered in $96.5 \%$ of all pregnancies (details in Table 1), and in $99 \cdot 2 \%$ (2177/2195) of the pregnancies that did not end in miscarriage, stillbirth, or voluntary termination $(98.5 \%$ in women $<25$ years $v s$. $99 \cdot 3 \%$ in older women, $P=0 \cdot 240$ ). ARV prophylaxis in infants also showed no difference by maternal age (97.2\% and $97 \cdot 3 \%$ in infants from younger and older women, respectively; OR $0.94,95 \%$ CI $0 \cdot 37-2 \cdot 39$, $P=0.895$ ). The main pregnancy outcomes, reported in Table 2, showed no major differences by age group. Among the pregnancies that ended in a live birth, the two age groups had similar rate of undetectable HIV viral load at third trimester $(58 \cdot 2 \%$ vs. $65 \cdot 2 \%$, Table 2). Given the possible role of some confounders in the significant differences observed, we performed two logistic regression analyses that analyzed the role of other potential relevant cofactors as predictors of pregnancy planned and HIV diagnosis in pregnancy. Such analyses confirmed the independent role of young age as a predictor of unplanned pregnancy and undiagnosed HIV infection before pregnancy. The details of univariate and multivariate analyses for 
Table 2. Pregnancy outcomes in the two age groups

\begin{tabular}{|c|c|c|c|c|c|}
\hline & Overall & Age lower than 25 & Age 25 or older & OR $(95 \% \mathrm{CI})$ & $P$ value* \\
\hline $\begin{array}{l}\text { HIV viral load }<50 \text { copies } / \mathrm{ml} \text { at } \\
\text { third trimester }(n: 1737), \% \text { : }\end{array}$ & $64 \cdot 6(1122 / 1737)$ & $58 \cdot 2(92 / 158)$ & $65 \cdot 2(1030 / 1579)$ & $0.74(0.53-1 \cdot 04)$ & $0 \cdot 080$ \\
\hline $\begin{array}{l}\text { Pregnancy not ending in a live } \\
\text { birth }(n: 2556), \%\end{array}$ & $13 \cdot 7(351 / 2556)$ & $12 \cdot 2(27 / 221)$ & $13 \cdot 9(324 / 2335)$ & $0 \cdot 86(0 \cdot 57-1 \cdot 31)$ & $0 \cdot 494$ \\
\hline $\begin{array}{l}\text { Pre-term delivery (<37 weeks) } \\
(n: 2175), \%\end{array}$ & $20 \cdot 4(443 / 2175)$ & $15 \cdot 8(30 / 190)$ & $20 \cdot 8(413 / 1985)$ & $0 \cdot 71(0 \cdot 48-1 \cdot 07)$ & $0 \cdot 102$ \\
\hline $\begin{array}{l}\text { Very pre-term delivery } \\
(<32 \text { weeks) }(n: 2175), \%\end{array}$ & $2 \cdot 4(53 / 2175)$ & $1 \cdot 6(3 / 190)$ & $2 \cdot 5(50 / 1985)$ & $0 \cdot 62(0 \cdot 19-2 \cdot 01)$ & $0 \cdot 426$ \\
\hline $\begin{array}{l}\text { Low birthweight }(<2500 \mathrm{~g}) \\
(n: 2096), \%\end{array}$ & $23 \cdot 3(489 / 2096)$ & $21 \cdot 7(39 / 180)$ & $23 \cdot 5(450 / 1916)$ & $0 \cdot 90(0 \cdot 62-1 \cdot 30)$ & $0 \cdot 581$ \\
\hline $\begin{array}{l}\text { Very low birthweight }(<1500 \mathrm{~g}) \\
(n: 2096), \%\end{array}$ & $3 \cdot 1(66 / 2096)$ & $1 \cdot 7(3 / 180)$ & $3 \cdot 3(63 / 1916)$ & $0 \cdot 50(0 \cdot 15-1 \cdot 60)$ & $0 \cdot 243$ \\
\hline $\begin{array}{l}\text { Small by gestational age } \\
(n: 1923), \%^{\dagger}\end{array}$ & $12 \cdot 2(235 / 1923)$ & $14 \cdot 3(24 / 168)$ & $12 \cdot 0(211 / 1755)$ & $1 \cdot 22(0 \cdot 77-1 \cdot 92)$ & $0 \cdot 393$ \\
\hline HIV transmission ( $n: 1685), \%$ & $1 \cdot 3(22 / 1685)$ & $2 \cdot 8(4 / 145)$ & $1 \cdot 2(18 / 1540)$ & $2 \cdot 40(0 \cdot 80-7 \cdot 18)$ & $0 \cdot 118$ \\
\hline Birth defects $(n: 2185), \%^{\star}$ & $3 \cdot 8(84 / 2185)$ & $4 \cdot 8(9 / 187)$ & $3 \cdot 8(75 / 1998)$ & $1 \cdot 30(0 \cdot 64-2 \cdot 63)$ & $0 \cdot 473$ \\
\hline
\end{tabular}

OR, odds ratio; $\mathrm{CI}$, confidence interval.

$* \chi^{2}$.

$\dagger$ Birthweight $<10$ th percentile by age and gender, singletons only.

+ Livebirths only.

Table 3. Factors associated with planning of pregnancy and diagnosis of HIV in pregnancy in univariate and multivariate analyses

\begin{tabular}{|c|c|c|c|c|c|c|}
\hline \multirow{2}{*}{$\begin{array}{l}\text { Outcome: planning of pregnancy } \\
\text { Predictive variables }\end{array}$} & \multicolumn{3}{|c|}{ Univariate analysis } & \multicolumn{3}{|c|}{ Multivariate analysis ( $n: 2443)$} \\
\hline & UOR & $95 \% \mathrm{CI}$ & $P$ value & AOR & $95 \% \mathrm{CI}$ & $P$ value \\
\hline Age (reference category $<25$ years) & $0 \cdot 50$ & $0 \cdot 36-0 \cdot 69$ & $<0 \cdot 001$ & $0 \cdot 60$ & $0 \cdot 43-0 \cdot 85$ & $0 \cdot 003$ \\
\hline Foreign origin & $0 \cdot 59$ & $0 \cdot 50-0 \cdot 70$ & $<0.001$ & $0 \cdot 69$ & $0 \cdot 58-0 \cdot 83$ & $<0 \cdot 001$ \\
\hline HIV undiagnosed before pregnancy & $0 \cdot 44$ & $0 \cdot 35-0 \cdot 55$ & $<0.001$ & $0 \cdot 52$ & $0 \cdot 41-0 \cdot 65$ & $<0 \cdot 001$ \\
\hline \multirow{2}{*}{$\begin{array}{l}\text { Outcome: diagnosis of HIV in pregnancy } \\
\text { Predictive variables }\end{array}$} & \multicolumn{3}{|c|}{ Univariate analysis } & \multicolumn{3}{|c|}{ Multivariate analysis ( $n: 2496)$} \\
\hline & UOR & $95 \% \mathrm{CI}$ & $P$ value & AOR & $95 \%$ CI & $P$ value \\
\hline Age (reference category $<25$ years) & $3 \cdot 29$ & $2 \cdot 54-4 \cdot 25$ & $<0 \cdot 001$ & 1.88 & $1 \cdot 35-2 \cdot 61$ & $<0 \cdot 001$ \\
\hline Foreign origin & $3 \cdot 88$ & $3 \cdot 21-4 \cdot 71$ & $<0 \cdot 001$ & $3 \cdot 53$ & $2 \cdot 79-4 \cdot 46$ & $<0 \cdot 001$ \\
\hline No preconception counseling & $35 \cdot 96$ & $20 \cdot 17-64 \cdot 10$ & $<0 \cdot 001$ & $30 \cdot 64$ & $16 \cdot 20-57 \cdot 95$ & $<0 \cdot 001$ \\
\hline Primigravida & $2 \cdot 44$ & $2 \cdot 03-2 \cdot 94$ & $<0.001$ & $3 \cdot 15$ & $2 \cdot 47-4 \cdot 02$ & $<0 \cdot 001$ \\
\hline Asymptomatic HIV disease & $5 \cdot 63$ & $3 \cdot 66-8 \cdot 65$ & $<0 \cdot 001$ & $4 \cdot 81$ & $2 \cdot 94-7 \cdot 87$ & $<0 \cdot 001$ \\
\hline
\end{tabular}

Univariate analyses, contingency tables with $\chi^{2}$ test; UOR, unadjusted odds ratio; CI, confidence interval; multivariate analysis, multivariable logistic regression models; AOR, adjusted odds ratio.

these two outcomes, with adjusted ORs for age and the other covariates are reported in Table 3.

\section{DISCUSSION}

This longitudinal study provided new findings on HIV and pregnancy in young women. First, we showed that within the population of pregnant women with HIV in Italy, the proportion of younger women is increasing. This finding is consistent with the high number of new HIV infections reported by WHO in young people [1], and with other local studies that reported a recent increase in the proportion of young pregnant women with HIV [8]. We also showed 
that, despite a positive temporal trend indicating increasing rates of planned pregnancy, a very high proportion of pregnancies $(63 \%)$ remain unplanned among women with HIV. National data are scarce, but the rate of planned pregnancy in the general population is likely to be higher [9]. In terms of predictors, younger age and foreign origin were two strong and independent determinants of both unplanned pregnancy and diagnosis of HIV in pregnancy. This significant role of younger age and foreign origin in pregnancy planning is consistent with the marked differences reported in fertility and abortion rates between Italian and foreign women [10], and with the higher rate of induced abortion observed in younger women with HIV [11]. Overall, these findings underline the urgent need to promote more efficiently HIV testing and reproductive counseling among young [12, 13] and migrant women [14].

It has already been shown that women with HIV are less likely to receive adequate and timely prenatal care compared with women without HIV [15]. The later entry in HIV care observed for younger women indicates an even more vulnerable subgroup within a population who already receives suboptimal care. Although the analysis of maternal treatment coverage and pregnancy outcomes showed no significant differences by maternal age in all the outcomes considered, younger women, being quite commonly unaware of their HIV infection status at booking in pregnancy, were less frequently on ARV treatment at conception, as already described, with a later entry in HIV care and treatment [8]. Given the strong consequential associations that link late maternal HIV diagnosis and later start of treatment to unsuppressed HIV viral load and mother-to-child transmission, a later entry in HIV care may potentially translate in some infants infected with HIV [8, 16], and should not be therefore underestimated. In this study, the number of infants vertically infected with HIV was very low, not allowing any conclusion, but younger women had slightly lower rates of viral load suppression at third trimester, and slightly higher rates of vertical transmission.

The present findings therefore confirm the urgency of implementing as much as possible the WHO recommendations, with particular attention to promoting and enhancing HIV testing before pregnancy among young women and among foreign women [1, 17]. Women's knowledge of their own HIV-positive status is a significant predictor of family planning [18], and there is evidence that women already aware of being infected before pregnancy have an earlier attendance of antenatal care, with potential significant benefits [19]. Although HIV testing in pregnancy is extremely relevant and effective, earlier interventions may provide additional benefits to young and foreign women in terms of family planning and engagement in care. Potentially relevant strategies include promotion of testing and antenatal counseling by cross-cultural phone counseling [20], wider and better implementation of contraception counseling in pre- and post-natal maternal care [14], immediate testing in the presence of HIV indicator diseases [21], and rapid HIV testing in outpatients belonging to vulnerable/at-risk populations [22].

\section{ACKNOWLEDGEMENTS}

The authors thank Cosimo Polizzi and Alessandra Mattei of the Istituto Superiore di Sanità in Rome, Italy, for providing technical secretarial for this study. No compensation was received for this contribution.

This work (currently not funded) was supported in the past by public research grants (ref.: H85E08000200005) from the Italian Medicines Agency (AIFA). The funder had no role in study design, data collection, data analysis, manuscript preparation, and/or publication decision. No funding was received for this work from any of the following organizations: National Institutes of Health (NIH); Wellcome Trust; and the Howard Hughes Medical Institute (HHMI). The corresponding author had full access to all the data in the study and takes responsibility for the integrity of the data and the accuracy of the data analysis.

\section{DECLARATION OF INTEREST}

None.

\section{ETHICS APPROVAL}

Ethics approval was obtained from the Ethics Committee of the I.N.M.I. Lazzaro Spallanzani in Rome (ref. deliberation 578/2001, 28 September 2001).

\section{REFERENCES}

1. World Health Organization. HIV and Adolescents: Guidance for HIV Testing and Counselling and Care for Adolescents Living with HIV: Recommendations for a Public Health Approach and Considerations for Policy-Makers and Managers. Geneva: World Health 
Organization, 2013 (https://www.ncbi.nlm.nih.gov/ books/NBK217962/). Accessed 23 January 2017.

2. Koenig LJ, et al. Young, seropositive, and pregnant: epidemiologic and psychosocial perspectives on pregnant adolescents with human immunodeficiency virus infection. American Journal of Obstetrics and Gynecology 2007; 197: S123-S131.

3. Lima YA, et al. HIV-1 infection and pregnancy in young women in Brazil: socioeconomic and drug resistance profiles in a cross-sectional study. BMJ Open 2016; 6: $\mathrm{e} 010837$

4. Floridia M, et al. Diagnosis of HIV infection in pregnancy: data from a national cohort of pregnant women with HIV in Italy. Epidemiology and Infection 2006; 134: 1120-1127.

5. Floridia M, et al. Pregnancy outcomes and antiretroviral treatment in a national cohort of pregnant women with HIV: overall rates and differences according to nationality. BJOG 2007; 114: 896-900.

6. Bertino E, et al. Neonatal anthropometric charts: the Italian neonatal study compared with other European studies. Journal of Pediatric Gastroenterology and Nutrition 2010; 51: 353-361.

7. Scheuerle A, Tilson H. Birth defect classification by organ system: a novel approach to heighten teratogenic signalling in a pregnancy registry. Pharmacoepidemiology and Drug Safety 2002; 11: 465-475.

8. Fatti G, et al. Adolescent and young pregnant women at increased risk of mother-to-child transmission of HIV and poorer maternal and infant health outcomes: a cohort study at public facilities in the Nelson Mandela Bay Metropolitan district, Eastern Cape, South Africa. South Africa Medical Journal 2014; 104: 874-880.

9. Krause EL. 'They just happened': the curious case of the unplanned baby, Italian low fertility, and the 'end' of rationality. Medical Anthropology Quarterly 2012; 26: 361-382.

10. Marchesi M. Reproducing Italians: contested biopolitics in the age of 'replacement anxiety'. Anthropology \& Medicine 2012; 19: 171-188.

11. Ammassari A, et al. Induced first abortion rates before and after HIV diagnosis: results of an Italian selfadministered questionnaire survey carried out in 585 women living with HIV. HIV Medicine 2013; 14: 31-39.
12. Kenny $\mathbf{J}$, et al. Pregnancy outcomes in adolescents in the UK and Ireland growing up with HIV. HIV Medicine 2012; 13: 304-308.

13. Echenique M, et al. Behaviorally and perinatally HIVinfected young women: targets for preconception counseling. AIDS Care 2017; 29: 372-377.

14. Lauria $\mathbf{L}$, et al. The effect of contraceptive counselling in the pre and post-natal period on contraceptive use at three months after delivery among Italian and immigrant women. Annali Istituto Superiore di Sanità 2014; 50: 54-61.

15. Ng R, et al. Adequacy of prenatal care among women living with human immunodeficiency virus: a population-based study. BMC Public Health 2015; 15: 514. doi: 10.1186/s12889-015-1842-y.

16. Momplaisir FM, et al. Time of HIV diagnosis and engagement in prenatal care impact virologic outcomes of pregnant women with HIV. PLoS ONE 2015; 10: e0132262. doi: 10.1371/journal.pone.0132262.

17. Musumari PM, et al. Prevalence and correlates of HIV testing among young people enrolled in non-formal education centers in Urban Chiang Mai, Thailand: a crosssectional study. PLoS ONE 2016; 11: e0153452. doi: 10.1371/journal.pone.0153452.

18. Habte D, Namasasu J. Family planning use among women living with HIV: knowing HIV positive status helps - results from a national survey. Reproductive Health 2015; 12: 41.

19. Gill MM, et al. The association between HIV status and antenatal care attendance among pregnant women in rural hospitals in Lesotho. Journal of Acquired Immune Deficiency Syndromes 2015; 68: e33-e38.

20. Taglieri FM, et al. Communication and cultural interaction in health promotion strategies to migrant populations in Italy: the cross-cultural phone counselling experience. Annali dell'Istituto Superiore di Sanità 2013; 49: 138-142.

21. Scognamiglio $\mathbf{P}$, et al. The potential impact of routine testing of individuals with HIV indicator diseases in order to prevent late HIV diagnosis. BMC Infectious Diseases 2013; 13: 473. doi: 10.1186/1471-2334-13-473.

22. Uccella I, et al. HIV rapid testing in the framework of an STI prevention project on a cohort of vulnerable Italians and immigrants. AIDS Care 2017; 29: 996-1002. 